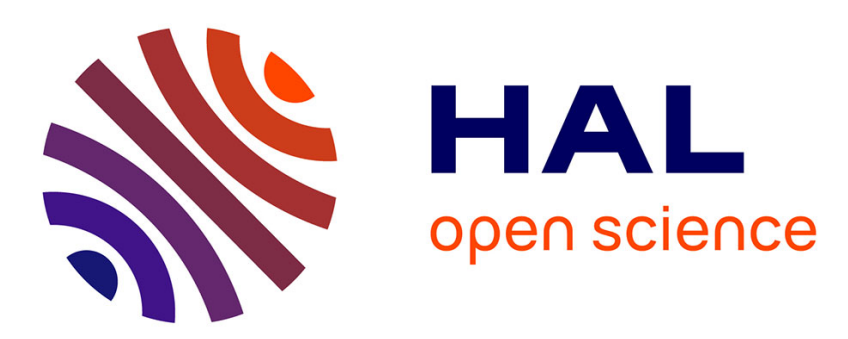

\title{
SEMICLASSICAL CALCULATION OF THE WIGNER TRANSFORM OF THE SINGLE PARTICLE DENSITY MATRIX AND SEMICLASSICAL HARTREE-FOCK
}

M. Durand, P. Schuck, J. Treiner

\section{To cite this version:}

M. Durand, P. Schuck, J. Treiner. SEMICLASSICAL CALCULATION OF THE WIGNER TRANSFORM OF THE SINGLE PARTICLE DENSITY MATRIX AND SEMICLASSICAL HARTREEFOCK. Workshop on Semiclassical Methods in Nuclear Physics, 1984, Grenoble, France. pp.C6-191C6-197, 10.1051/jphyscol:1984622 . jpa-00224223

HAL Id: jpa-00224223

https://hal.science/jpa-00224223

Submitted on 1 Jan 1984

HAL is a multi-disciplinary open access archive for the deposit and dissemination of scientific research documents, whether they are published or not. The documents may come from teaching and research institutions in France or abroad, or from public or private research centers.
L'archive ouverte pluridisciplinaire HAL, est destinée au dépôt et à la diffusion de documents scientifiques de niveau recherche, publiés ou non, émanant des établissements d'enseignement et de recherche français ou étrangers, des laboratoires publics ou privés. 


\title{
SEMICLASSICAL CALCULATION OF THE WIGNER TRANSFORM OF THE SINGLE PARTICLE DENSITY MATRIX AND SEMI CLASSICAL HARTREE-FOCK
}

\author{
M. Durand, P. Schuck and J. Treiner ${ }^{\star}$ \\ Institut des Sciences Nucléaires, 53, Avenue des Martyrs, \\ 38026 Grenoble Cedex, Franee \\ *Institut de Physique Nucléaire, Division de Physique Théorique, \\ B.P. $n^{\circ}$ 1, 91406 Orsay, Fronce
}

\begin{abstract}
Résumé - Nous montrons comment la partie moyenne de la transformée de Wigner de $\rho$ correspondant à un potentiel Woods-Saxon peut être calculée semi-classiquement. Nous trouvons une diffusivité dans 1 'espace de phase appréciable mais toujours bien plus petite que l'énergie de Fermi, ce qui. justifie le développement en $\hbar$ à la Wigner-Kirkwood. En utilisant ce fait, nous proposons et réalisons une nouvelle méthode semi-classique pour le problème Hartree-Fock qui est directement basée sur le développement en ऋ de Wigner-Kirkwood. En conséquence, nous n'avons pas besoin d'une fonctionnelle $\tau[\rho]$.

Abstract - It is shown how the smooth part of the Wigner transform of $\rho$ corresponding to a Woods-Saxon potential can be calculated semiclassically. We find that its surface thickness in phase space is appreciable but always much smaller than the Fermi. energy thus justifying the WignerKirkwood T expansion. Using this fact we propose and perform a new semiclassical Hartree-Fock method directly based on the Wigner-Kirkwood expansion. No functional $\tau[\rho]$ is therefore needed.
\end{abstract}

At first we want to study the Wigner transform of a density matrix corresponding to A independent nucleons in a phenomenological potential like e.g. the Woods-Saxon potential.

It is well known how to obtain the usual Wigner Kirkwood h expansion for the Wigner transform of the density matrix :

$$
\begin{aligned}
f(\vec{R}, \vec{p})= & \left\{\theta\left(\varepsilon_{F}-\hat{H}\right)\right\}_{W}=\theta\left(\varepsilon_{F}-H_{C}\right)+\frac{1}{2} \delta^{\prime}\left(\varepsilon_{F}-H_{c}\right)\left[\left(\hat{H}-H_{c}\right)^{2}\right]_{W} \\
& +\frac{1}{6} \delta^{\prime \prime}\left(\varepsilon_{F}-H_{C}\right)\left[\left(\hat{H}-H_{C}\right)^{3}\right]_{W}+\ldots
\end{aligned}
$$

Here $W$ stands for Wigner transform, $\hat{H}$ is the șingle particle Hamiltonian and $H_{c}$ its classical counterpart.

The Wigner transforms of the different powers of $\left(\hat{\mathrm{H}}-\mathrm{H}_{\mathrm{c}}\right)$ are easily evaluated and one obtains in the case of a local potential $V(\vec{R})$ up to order $\pi^{2} / 1 /$ (generalisation to non local potential is straightforward) :

$$
\begin{aligned}
f(\vec{R}, \vec{p})= & \theta\left(\varepsilon_{F}-H_{c}\right)-\frac{1}{4} \frac{\hbar^{2}}{2 m} \Delta V \delta^{\prime}\left(\varepsilon_{F}-H_{c}\right) \\
& +\frac{1}{12} \frac{\hbar^{2}}{2 m}\left[(\vec{\nabla} V)^{2}+(\vec{p} \cdot \vec{\nabla})^{2} V / m\right] \delta^{\prime \prime}\left(\varepsilon_{F}-H_{c}\right)+\sigma\left(\hbar^{4}\right)
\end{aligned}
$$


This expansion is evidently an asymptotic expansion in terms of distributions where the derivatives of $\delta$-functionsmock up a certain surface thickness to be added to the pure step function of the lowest order (Thomas-Fermi) term.

A very similar expansion exists for the low temperature expansion of the Fermi distribution $/ 2 /$ :

$$
F(\varepsilon)=\left[1+\exp \frac{\varepsilon-\varepsilon_{F}}{T}\right]^{-1} \simeq \theta\left(\varepsilon_{F}-\varepsilon\right)+\frac{\pi^{2}}{6} T^{2} \delta^{\prime}\left(\varepsilon_{F}-\varepsilon\right)+\cdots
$$

Al so here the $\delta^{\prime}$-term simulates the finite diffuseness of the Fermi function as can be easily verified in "enlarging" the $\delta$-function somewhat. The formula (3) is an asymptotic expansion since $F(\varepsilon)$ has an essential singularity in $T$ and thus does not allow for a Taylor series in $T$ in the ordinary sense. Nevertheless (3) is for $T<\varepsilon_{\mathrm{F}}$ extremely precise for the evaluation of mean values since one can show (from the derivation of eq. (3)) that the error is of order exp $\left(-\varepsilon_{\mathrm{F}} / \mathrm{T}\right)$. To assess whether (2) is also an accurate asymptotic expansion of the true function we have to $f$ ind the function to which (2) is its asymptotic expansion (it is well known (s. harmonic oscillator) that the Wigner transform of the true density matrix has an essential singularity in $\pi$ and thus $\hbar$ in (2) plays an analogous role to $T$ in (3)). This reconstitution is probably only possible approximately; we want to do this in the following way.

The Laplace transform with respect to the variable $\varepsilon_{F}$ is given by $/ 1 /$ :

$$
\begin{aligned}
C^{\beta}(\vec{R}, \vec{\varphi})=\beta & \mathcal{L}_{\varepsilon_{F} \rightarrow \beta} f(\vec{R}, \vec{p})=e^{-\beta H_{c}}\left\{1+\frac{\hbar^{2} \beta^{2}}{8 m}[-\Delta V+\right. \\
& \left.\left.+\frac{\beta}{3}(\vec{\nabla} V)^{2}+\frac{\beta}{3 m}(\vec{p} \cdot \vec{\nabla})^{2} V\right]+\sigma\left(\hbar^{4}\right)\right\}
\end{aligned}
$$

One can show $/ 1 /$ that all powers of $\overrightarrow{\nabla V}$ in (4) are partially resummed if one writes the $(\vec{\nabla} V)^{2}$ term explicitely shown in (4) into the exponent :

$$
\begin{aligned}
C^{\beta}(\vec{R}, \vec{\varphi})= & \exp \left[-\beta H_{c}+\frac{\hbar^{2} \beta^{3}}{24 m}(\vec{\nabla} v)^{2}\right] \\
& \left\{1+\frac{\hbar^{2} \beta^{2}}{8 m}\left[-\Delta V+\frac{\beta}{3 m}(\vec{p} \cdot \vec{\nabla})^{2} V\right]+\cdots\right\}
\end{aligned}
$$

This expression is incidentally exact for a linear potential. We should however notice that for a general potential (5) represents a local approximation and is thus only valid in the short time limit; in order to eliminate the spurious long time behavior we multiply (5) by a Strutinsky smooțhing factor $f_{M}$ (BY) /1, p. 539/ (the usual technique to handle this problem, namely Laplace inversion by saddle point method turns out to be not accurate enough here); the Laplace inversion is then performed numerically what is of no major problem because the Strutinsly factor cuts the $\beta$ - integration off.

We did this procedure for a harmonic oscillator potential (3 dim., spherical) and took $\gamma \simeq 2.5 \hbar \omega_{0}$; this corresponds to the value taken indepently for a pure Strutinsky calculation of $f$ and which turned out to be the adequate value to damp out all shell effects $/ 3 /$. For a harmonic potential with closed shells the wigner function depends on the classical energy only. In Fig. 1 we represent our results together with the Strutinsky calculation (indistinguishable on the drawing), the ThomasFermi, and exact results $(A=224)$ Our "reconstitution" of the exact function from the series /2/ contains of course no shell effects but the fact that the 1ast bump (Friedel oscillation) before the descent is reproduced exactly as in the strutinsky calculation is, we think, a remarkable feature. It means nothing else than that the expansion (2) contains this subtlety demonstrating again the high degree of accuracy 


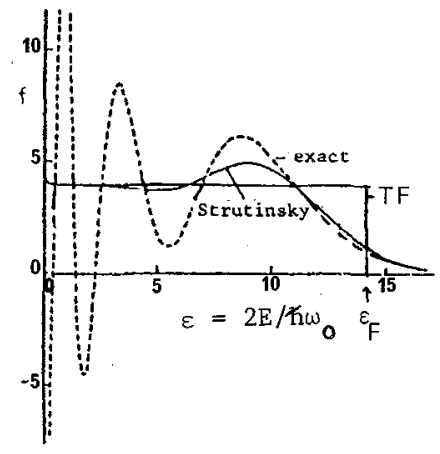

Fig. 1 - Representation of the exact, Strutinsky and semiclassical (indistinguishable on the graph). Wigner transform of $\rho$ for $A=224$ in $a$ spherical harmonic oscillator. The pure Thomas-Fermi result is also indicated. The results are shown as a function of the reduced variable $\varepsilon=2 \mathrm{E} / \varkappa_{\mathrm{o}}$ o with $\mathrm{E}=\mathrm{H}_{\mathrm{c}}$.

of the Wigner kirkwood expansion (2). We can now consider the expansion (2) as the precise asymptotic series to the Strutinsky smoothed Wigner distribution $f$ and also our validity criterion of the expansion (3) for the Fermi function holds : the diffusivity, though quite large, is still considerably smaller than the Fermi energy and as a matter of fact, fitted one crudely a Fermi function to the distribution in Fig.1, a diffusivity parameter of $a \simeq 4 \mathrm{MeV}$ would be found. This should be compared to the Fermi energy $\varepsilon_{\mathrm{F}} \simeq 40 \mathrm{MeV}$ such that the exponentially small error contained in (2) with respect to the true smooth function is : $\exp \left(-\varepsilon_{\mathrm{F} / \mathrm{a}}\right) \simeq \exp (-10) \simeq$ $10^{-4}-10^{5}$. This error is indeed very small and explains the complete numerical agreement between Strutinsky and Extended Thomas-Fermi calculations which has always been found /4/. In complete analogy to what has been done in the case of the real nuclear density we therefore want to call (2) the leptodermous expansion $/ 5 /$ of $\mathrm{f}$ in phase space.

The Wigner distribution function for nucleons in a spherical Woods Saxon potential is more complicated because it depends not only on $\mathrm{p}^{2}$ like in the harmonic oscillator case but also on $\vec{p} \cdot \vec{R}$. For an easier graphical display we averaged therefore over all direction of $\vec{p}$ (see however ref. $/ 6 /$ ); furthermore the $\mathrm{R}$, p-dependence can. not be put into the one variable which is the classical energy; nevertheless we took as one variable again the reduced harmonic oscillator energy $\varepsilon=2 \mathrm{H}_{\mathrm{C}} \mathrm{Ho} / \mathrm{h} \omega_{\mathrm{O}}$ ( $* \omega_{0}=41 \mathrm{~A}^{-1 / 3}$ ) and as a second variable $\theta=\operatorname{arctg}(\mathrm{p} / \mathrm{R})$ which thus represent sort of polar coordinates in phase space. A more delicate problem constitutes the choice of $\gamma$ in the strutinsky smearing function $f_{M}(B \gamma)$. We took it proportional to the absolute value of the local curvature of the potential and introduced thus a $R-$ dependent $\gamma$. However for regions where the local curvature dropped below $h \omega_{0}$ (the corresponding harmonic oscillator value) we took $\gamma \simeq$ t $\omega_{0}$. This somewhat ad hoc prescription turned out to be appropriate in order to reconstitute most accurately the true Wigner distribution to which (2) is its asymptotic expansion.

In Fig. 2 we display our semiclassical result of the Wigner transform of $\rho$. Preliminary studies show that the corresponding Wigner transform of the exact $\rho$ agrees on the average rather well with the surface part (in phase space) of the semiclassical values. As before the quantum oscillations in the interior are however completely washed out in the semiclassical calculation.

The reconstitution of the phase space distribution has been performed in order to visualize what is behind the expansion (2) and to assess its accuracy; for many actual calculations however we can perfectly use eq. (2) and as a matter of fact it is preferable to use (2) instead of the reconstruction because it is easier to handle and at the same time more accurate. The latter point comes from the fact that the partial resummation (5) of the gradient terms destroys the order by order consistency in $\hbar$ inherent to eq.(2). For some problems it is nevertheless very important to know the behavior of $f$ in phase space one of which is the Hartree-Fock problem to which we want to turn now. 
The understanding of the meaning of eq.(2) as a leptodermous expansion in phase space leads namely directly to a novel solution of the semiclassical Hartree-Fock problem. This needs no knowledge of a functional $[\rho]$ and makes directly use of the fast convergence of (2) (faster than the functional $\tau[\rho]$ where one has to go to fourth order /7/ whereas (2) is usually converged after the $\pi^{2}$-term).

The very simple procedure is as follows : we have to find a parametrisation of $f(R, p)$ such as shown in Fig.2; with this trial $f_{t}$ we construct the Wigner transform of the non local Hartree-Fock field VH.F. $(R, p)$ and insert it into the non local. generalisation of eq. (2); we find a corresponding Wigner Kirkwood $f_{W . K}$. and the parameters of $f_{t}$ are found in adjusting a number of moments of $R$ and $p$ such that

$$
\int d \Gamma \sigma_{n}(R, p) f_{W \cdot K}=\int d \Gamma \sigma_{n}(R, p) f_{t} ; d \Gamma=\frac{d^{3} R d^{3} p}{(2 \pi \hbar)^{3}}
$$

where $\sigma_{n}$ can be any power of $R$ and $p$ (also fractional powers) or any function of $R$ and $p$ such as $R \cdot p$ etc. The number $n$ must of course be equal to the number of parameters necessary for $f_{t}$. As we will show this procedure is variational and will thus depend little on the specific set of functions $\sigma_{n}$ but still one should check the convergence of the procedure as a function of $n$.

Instead of adjusting moments it would also be feasible to calculate the groundstate energy with $f_{W . K}$. depending on the parameters via $\mathrm{V}^{\mathrm{H}} \cdot \mathrm{F} \cdot(\mathrm{R}, \mathrm{p})$ and $\mathrm{f}_{\mathrm{t}}$ and minimise. We instead want to show here that the procedure (6) is variational.

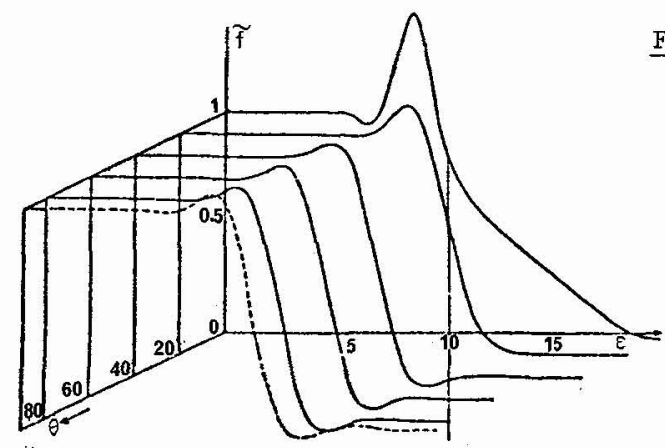

Fig. 2 - Smooth wigner distribution $\widetilde{\mathrm{f}}(\varepsilon, \theta), \varepsilon=2 \mathrm{H}_{\mathrm{c}} \mathrm{HO} / \mathrm{h} \omega_{\mathrm{O}}$ $\theta=\operatorname{artg} p / R$ for a Woods Saxon potential $\left(\mathrm{V}_{0}=-50 \mathrm{MeV}\right.$ $\mathrm{R}=7.3 \mathrm{fm} \quad \mathrm{a}=0.6 \mathrm{fm}$ $A=224$, usual notation). The twiggle indicates that we averaged over all direction of $\vec{p}$ in order to allow for a convenient graphical representation as a function of two variables only.

The variational principle for Slater determinants can be cast into the form

$$
\delta\left\{\operatorname{Tr}(t \rho)+\frac{1}{2} \operatorname{Tr} \operatorname{Tr} \rho \bar{v} \rho-\operatorname{Tr} \Omega\left(\rho^{2}-\rho\right)\right\}=0
$$

where the matrix $\Omega$ of Lagrange multipliers assures that the single particle density matrix corresponds to a slater determinant. The meaning of all other terms in (7) is self evident. The variational principle (7) leads to the following equation

$$
\left[h^{\text {H.F. }}, \rho\right]=\Omega\left(\rho^{2}-\rho\right)
$$

Equation (8) is usually solved with $h^{\text {H.F. }}|i\rangle=\varepsilon_{i}, \mid i>$ where the single particle states $\mid i>$ are used to construct $\rho$; self consistency is achieved when $\rho$ entering $\mathrm{h}^{\mathrm{H}}$.F. is the same as the $\rho$ constructed from $|i\rangle$. This procedure assu- 


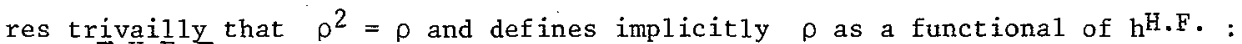
$\rho=\rho\left[\mathrm{h}^{\mathrm{H}} \cdot \mathrm{F} \cdot\right]$. This functional cannot be given explicitly in general but in (2) as a special case the functional is known. It is well known /8/ and easy to verify that this functional solves $\left[\mathrm{hH}^{\mathrm{H} . \mathrm{F}}, \rho, \rho\right]=0$ order by order in $k$. It therefore remains to show that also $\rho^{2}=\rho$ is verified to each order in $\hbar$ (to lowest order this is of course trivially the case since $\theta^{2}=\theta$ ). To this purpose we take the Wigner transform of $\rho^{2}=\rho$ and obtain with $\rho=\rho_{0}+\hbar^{2} \rho_{2}$ to second order in $\hbar$ :

$$
\rho_{2 w}\left(2 \rho_{0 w}-\Lambda\right) \stackrel{?}{=} \frac{1}{8} S_{0 w} \wedge^{2} \rho_{0 w}
$$

where $\Lambda=\vec{\nabla}_{R} \dot{\vec{\nabla}}_{p}-\bar{\nabla}_{p} \vec{\nabla}_{R}$ is the classical operator defining the Poisson bracket. Equation ( 9 ) is of course a quite subtle relation since as we see from (2) it involves products of distributions; for their evaluation we first consider (9) at finite temperatures and let $T$ go to zero at the end. We thus have

$$
\rho_{\mathrm{W}} \rightarrow\left[i+\exp \frac{h_{c}-\varepsilon_{F}}{T}\right]^{-1}:=F\left(\varepsilon_{F}\right) ; \quad \delta^{\prime}\left(\varepsilon_{F}-h_{c}\right) \rightarrow \frac{\partial^{2}}{\partial \varepsilon_{F}^{2} F} ; \text { etc. }
$$

Inserting (10) into (9), using (2) for $\rho_{2}$, explicitly performing al1 derivatives on the Fermi function (what introduces higher powers of $F$ ), and then taking the limit $\mathrm{T} \rightarrow 0$, we find that (9) is verified (order $\pi^{2}$ ). We thus conjecture that $\rho^{2}=\rho$ holds oxder by order in $\hbar$.

This leads us to the very gratifying result that the semiclassical functional (2) $f(\vec{R}, \vec{p})=f\left[h^{H} \cdot F \cdot\right]$ is solution of the variational equation (8).

We applied this to a simple model case in using two skyrme forces SIII and SVII for symmetric $(\mathrm{N}=\mathrm{Z})$ systems neglecting spin orbit and Coulomb terms. For Skyrme forces our problem is simplified since only local quantities are needed. We thus have to parametrise only the density and not the full phase space distributions. Semiclassical (1iquid drops) densisities are Fermi function-1ike and one can show that

$$
\rho(r)=\left(1+c_{1} \frac{\partial}{\partial r}+c_{2} \frac{\partial^{2}}{\partial r^{2}}+\cdots\right) F(r)
$$

with $F(r)$ a Fermi function is a systematic expansion of the semiclassical density; we actually kept only the linear term in (11) which amounts to represent $\rho(r)$ as a superposition of $F$ and $F^{2}$, i.e. $\rho(r)=\alpha_{1} F+\alpha_{2} F^{2}$. The four parameters can be fixed with (6) in choosing $o_{n}=r^{n}$ with $n=-1,0,1,2$. Actually there is one further parameter $\varepsilon_{F}$ contained in (2) which is as usual determined from the particle number condition.

Our semiclassical results obtained in this way are of course to be compared with self-consistent Strutinsky Hartree-Fock. (HFS) calculations what we do for a number of cases in table 1. (We are graterul to $\mathrm{Dr} \mathrm{J}$. Bartel for providing us with the HFS results).

We see on the whole excellent agreement between semiclassical (W.K.)and HFS results.It has been noticed before $/ 9 /$ that average densities are actually closer to $\mathrm{F}^{2}$ than to F. This subtlety is very well reproduced in our study confirming once again that fine details are contained in (2). In Fig. 3 we also compare our semiclassical density with the exact one and see that good agreement on the average is achieved.

A further delicate test for our theory is the determination of the level density parameter $g\left(\varepsilon_{\mathrm{F}}\right)$ at the Fermi surface. It can be directly calculated (without making the detour over a low temperature expansion) from (2) with 


$$
g\left(\varepsilon_{F}\right)=\frac{\partial}{\partial \varepsilon_{F}} \int \frac{d^{3} R d^{3} p}{(2 \pi \hbar)^{3}} f(\vec{R}, \vec{p})
$$

\begin{tabular}{|c|c|c|c|c|}
\hline \multicolumn{2}{|l|}{ A } & 40 & 140 & 184 \\
\hline & $\mathrm{HF}$ & -397.3 & -1649.6 & -2246.9 \\
\hline $\mathrm{E}_{\text {tot }}[\mathrm{MeV}]$ & $\mathrm{HFS}$ & -382.1 & & -2230.7 \\
\hline S III & WK & -384.2 & -1642.7 & -2225.3 \\
\hline$\left\langle r^{2}\right\rangle$ & $\mathrm{HF}$ & 3.37 & 4.89 & 5.33 \\
\hline$S$ III & WK & 3.41 & 4.93 & 5.37 \\
\hline$\alpha_{1} / \alpha_{2}$ & WK & 0.11 & 0.015 & 0.003 \\
\hline & $\mathrm{HF}$ & -397.1 & -1649.2 & -2243.4 \\
\hline$E_{\text {tot }}[\mathrm{MeV}]$ & $\mathrm{HFS}$ & & -1648.5 & -2238.4 \\
\hline S VII & WK & -388.0 & -1651.1 & -2237.4 \\
\hline$\left\langle\mathrm{r}^{2}\right\rangle$ & $\mathrm{HF}$ & 3.38 & 4.90 & 5.35 \\
\hline S VII & WK & 3.38 & 4.92 & 5.36 \\
\hline$\alpha_{1} / \alpha_{2}$ & WK & 0.10 & 0.004 & 0.01 \\
\hline
\end{tabular}

Table 1

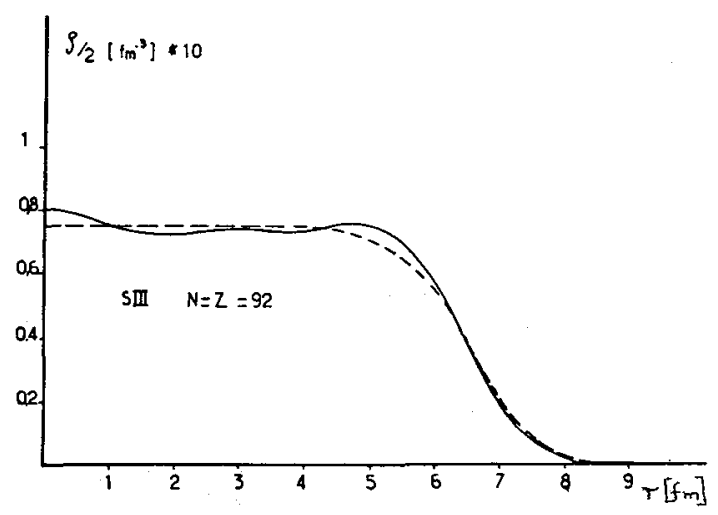

Fig.3 - Exact Hartree-Fock density (ful1 1ine) and semiclassical density (broken line) for $N=Z=92(S$ III $)$.

We find for the case $\mathrm{A}=184$ ( $\mathrm{S}$ III) $\mathrm{g}=8.58$ to be compared with the HFS result of $g=8.52$. This shows that also for this quantity our theory gives very satisfactory results. Notice that usually calculations using the density functional method are about 20 to $30 \%$ away from the HFS result though Brack and collaborators /10/ 
seem to have overcome this difficulty. On the other hand it seems impossible within the density functional method to calculate such quantities as $\partial / \partial \varepsilon_{\mathrm{F}} \mathrm{g}\left(\varepsilon_{\mathrm{F}}\right)$ because of a diverging result whereas in the Wigner Kirkwood method this is perfect $1 \mathrm{y}$ possible.

As a last point we would like to mention that our theory can easily be generalised to finite temperatures. We only have to make the same expansion as in eq. (1) for the temperature dependent density matrix

$$
f_{T}(\vec{R}, \vec{p})=\left\{1+\exp \frac{\hat{H}-\varepsilon_{F}}{T}\right\}_{W}^{-1}
$$

All step and $\delta$-functions in (2) have then to be replaced by $\theta\left(\varepsilon_{F}-H_{C}\right) \rightarrow F\left(H_{C}\right)$, $\delta^{\prime}\left(\varepsilon_{\mathrm{F}}-\mathrm{H}_{\mathrm{C}}\right) \rightarrow \partial^{2} \mathrm{~F} / \partial \varepsilon_{\mathrm{F}}{ }^{2}$, etc. It should be noticed that for temperatures $\mathrm{T}>3-4 \mathrm{MeV}$ where all shell effects are washed out our method should essentially coincide with the exact H.F. method.

In conclusion we can say that in conjunction with a quite precise reconstitution of the Wigner distribution to which the usual k-expansion is its asymptotic expansion we have obtained a very clear picture of the meaning and the validity of our semiclassical appraoch : the Wigner Kirkwood fi-expansion constitutes a leptodermous expansion in phase space of the true average distribution which indeed has a surface thickness in phase space (which can be characterised globally by diffusivity parameter of $a \simeq 4 \mathrm{MeV}$ ) quite small compared to the Fermi energy. Because of the exponentially small error involved in the asymptotic expansion we can assess the error to be of the order $10^{-4}-10^{-5}$ in complete agreement with the accord which has always been found between semiclassical and Strutinsky calculations.

From these considerations we deduce a novel method for the semiclassical HartreeFock problem. It uses directly the Wigner-Kirkwood expansion and does not have to make the laborious and somewhat undefined detour over a density functional

$\tau[\rho]$. The simplicity of our approach allows us to envisage the use of finite range forces and inclusion of pairing and finite temperatures.

\section{REFERENCES}

/1/ RING P., SCHUCK P., The Nuclear Many Body Problem, Springer-Verlag, 1980

/2/ CHANDRASEKHAR S., An introduction to the study of stellar structure (Dover, N.Y. 1967) 2nd Edit. (1st Edit. 1939)

/3/ PRAKASH M. , SHLOMO S., KOLOMIETZ V.M., Nucl. Phys. A 370 (1981) 30

/4/ JENNINGS B.K., Ph. D. Thesis, Mc Master University (1976)

/5/ SCHUCK P., Lecture Notes held at school on "Density Functional Methods in Physics", Sept. 5-11, 1983 Alcabideche (Portugal)

16/. DURAND M., RAMAMURTHY V.S., SCHUCK P., Phys. Lett. 133B (1982) 116

/7/ GUET C., BRACK M., Z. Physik A 297 (1980) 247

/8/ CARRUTHERS P., ZACHARIASEN F., Rev. Mod. Phys. 55 (1983) 245

/9/ TREINER J., Thèse d'Etat, Orsay 1981

/10/GUET C., BRACK M., to be published. 